\title{
ESTUDIO SOBRE ALGUNOS TREMELLALES DE MEXICO
}

\author{
por \\ B. Lowy*
}

En este trabajo se presenta un resumen taxonómico de los hongos tremeláceos de México, hasta ahora no estudiados en este país. Además, hay pocos estudios que tratan de los Tremellales de América Latina y referencias a ellos en la literatura son escasas aún (Lowy 12, 13; Martin, 15, 16, 17, 18, 21; Moeller, 22; Patouillard y Lagerhaim, 24; Viegas, 28). Como esta investigación puede servir de introducción a un grupo de Basidiomycetes todavía poco conocido en México, conviene presentar claves de los órdenes, familias, géneros y especies, junto con notas breves sobre cada especie. Aunque los hongos tremeláceos tienen poca importancia económica, porque casi todos son saprófitos [pero véanse Herrera y Guzmán, (4) sobre especies comestibles de Auricularia], son de mucho interés morfológico por la gran variación en la estructura del basidio. Hay entre ellos, algunos que tienen basidios cilíndricos, ovoides o fusiformes, septados o unicelulares. Dentro del mismo género, y a veces en la misma especie, el número de septas en el basidio es variable (Lowy, 10). Esta inestabilidad morfológica se debe considerar como un carácter importante de un estado primitivo en el desarrollo del basidio. Por otro lado, el basidio de los Homobasidiomycetidae es mucho más estable en su estructura, lo cual indica su posición avanzada en la escala evolutiva. Por estas razones, entre otras, hay distintas interpretaciones filogenéticas de los Tremellales encontrados en los trabajos de Donk (2,3), Linder (6), Martin (14, 20), Neuhoff (23) y Rogers (25).

Desde mediados de junio hasta fines de agosto de 1962, me dediqué al presente estudio mientras contaba con la valiosa ayuda del Dr. Teófilo Herrera del Instiuto de Biología, quien me ofreció todas las facilidades del Instituto y de su laboratorio. Quiero agradecer mucho también al Dr. M. Ruiz Oronoz, del Jardín Botánico de la U.N.A.M. y a los profesores Gastón Guzmán y Marta Zenteno, quienes me acompañaron en algunos viajes al campo en busca de especímenes. El Dr. J. P. Ojeda, Director del Instituto Tecnológico Azucarero Veracruzano, facilitó mucho mis viajes a Veracruz, a Tahasco y a Chiapas y le estoy muy agradecido por sus numerosas cortesías.

Se pueden identificar los Tremellales, conocidos comúnmente bajo el nombre de hongos gelatinosos, según la clave siguiente.

Hongos que, en el estado sexual, producen hasidios, los cuales llevan hasidiosporas $\ldots . . . \ldots . .$.

Clase BASIDIOMYCETES.

* Trabajo realizado con la ayuda del Council on Research, Louisiana State University y patrocinado por la American Philosophical Society. 
BOLETIN DE LA SOCIEDAD BOTANICA DE MEXICO, No. 29

Basidios maduros septados en 2 ó 4 células, o si unicelulares, bifurcados (Da. crymycetaceae); esterigmas (epibasidios) ${ }^{1}$ infladas ..... Subclase HE. TEROBASIDIOMYCETIDAE.

Basidio arpos en general bien diferenciados y conspicuos, frecuentemente gelatinosos, pero a veces inconspicuos y resupinados y de textura seca o cerosa; basidiosporas germinando comúnmente por .repetición ${ }^{2}$ Orden TREMELLALES

\section{CLAVE DE LAS FAMILIAS PRINCIPALES ENCONTRADAS EN MEXICO}

1 Basidios unicelulares y bifurcados cuando maduros, con dos esterigmas en forma de horquilla .........

DACRYMYCETACEAE.

1 Basidios tabicados en la madurez

2 Basidios cilíndricos con tabiques transversales ...... AURICULARIACEAE.

2 Basidios subglobosos o piriformes con tabiques comúnmente en forma de cruz (cruciado-septado) cuando maduros. ........ TREMELLACEAE.

Después de las claves de los géneros (enumerados) y de las especies, siguen unas breves notas sobre cada especie junto con la lista de colecciones estudiadas. En la enumeración del material estudiado, los nombres de los colectores principales son abreviados según la manera siguiente: Gastón Guzmán (GG), Teófilo Herrera (TH), M. Ruiz Oronoz (MRO), B. Lowy (BL). Las colesciones están depositadas en el Herbario Nacional del Instituto de Biología de la U.N.A.M. (MEXU) y en el Herbario Micológico de la Universidad de Louisiana (LSUM).

\section{CLAVE DE LOS GENEROS DE DACRYMYCETACEAE}

1 Fructificación erecta, delgada, sencilla o ramificada, formando cuernitos ana. CALOCERA (J)

1 Fructificación pulvinada, efusa o estipitada

2 Fructificación estipitada y espatulada o flabeliforme en la parte superior DACRYOPINAX

1 Según algunos micólogos, el t-rmino "epibasidio" debiera llamarse "esterigma", mientras otros prefieren usar el término "espiculo" por la estructura que lleva las esporas. Talbot (26) y Donk (2) han presentado la mejor exposición de esta terminología.

2 La formación de un esterigma en una basidiospora, sobre la cual se forma una espora secundaria. Este fenómeno se puede repetir varias veces. 
2 Fructificación nunca espatulada o flabeliforme en la la parte superior

3 Distintamente estipitada, la parte superior hinchada o morcheliforme

3 Pulvinada, efusa o subestipitada, con himenio liso, giroso

o cerebriforme .... 4

4. Efusa, con textura cerosa, nunca estipitada ......ARRHYTIDIA (IV)

4. Pulvinada o subestipitada, himenio giroso o cerebriforme

\section{CLAVE DE LAS ESPECIES DE DACRYOPINAX}

1 Basidiocarpo espatulado-alargado en la parte superior; de color anaranjado-amarillento cuando fresco (mojado), y más ocuro seco; esporas uniseptadas

D. spathularia

1 Basidiocarpo estipitado y flabeliforme en la parte superior; esporas triseptadas

D. elegans

\section{CLAVE DE LAS ESPECIES DE DACRYMYCES}

1 Basidiocarpo cerebriforme o giroso, casi siempre de

$1 \mathrm{~cm}$. o más de largo

1 Basidiocarpo pulvinado, con pocas circunvoluciones y en general, menos de $0.55 \mathrm{~cm}$. de largo

2 Esporas triseptadas ......... ellisii

2 Esporas uni-heptaseptadas

3 Esporas transversalmente heptaseptadas y con varias septas verticales ......... D. dictyosporus

3 Esporas heptaseptadas pero sin septas verticales D. palmatus

4. Basidiocarpo al secar aplanado e inconspicuo; hifas con hebillas; esoras triseptadas, las septas en general gelatinosas

D. deliquescens.

4. Basidiocarpo, al secar más conspicuo, arrugado, con aspecto de pequeñas pasas de uvas; hifas sin hebillas; esporas triseptadas

......... punctiformis 


\section{CLAVE DE LOS GENEROS DE AURICULARIACEAE}

1 Fructificación pileada, aplanada o subestipitada, más o menos auriforme y hasta varios cms. de largo; abhimenio piloso ......AURICULARIA (VI)

1 Fructificación resupinada, efusa, de pocos $\mathrm{mm}$. de largo; sin pelos

\section{CLAVE DE LAS ESPECIES DE AURICULARIA}

1 Basidiocarpo con himenio diferenciado en forma de red conspicua; gelatinoso cuando fresco A. delicata

1 Basidiocarpo gelatinoso cuando fresco y con himenio liso; si el himenio tiene venas, el basidiocarpo no es gelatinoso cuando fresco $\ldots \ldots \ldots \ldots 2$

2 Corte transverso del basidiocarpo con una banda central de hifas más o menos densas y paralelas (médula) ......... 3

2 Corte transverso del basidiocarpo sin médula A. auricula

3 Abhimenio densamente piloso, con pelos en zonas distintas y concéntricas

........... mesenterica

3 Abhimenio sin zonas pelosas concéntricas

4. Pelos del abhimenio largos, cerca de 450 micras, médula de más de 100 micras de ancho A. polytricha

4. Pelos del abhimenio cortos, cerca de 500 micras; médula de menos de 75 micras de ancho

\section{CLAVE DE LOS GENEROS DE TREMELLACEAE}

1 Fructificación pileada, erecta, el himenio sobre espinas de hasta $5 \mathrm{~mm}$. de largo PSEUDOHYDNUM (VIII)

1 Fructificación con himenio liso, cerebriforme o folioso (con espinas de menos de $1 \mathrm{~mm}$. de largo en Eichleriella spinulosa)

2 Fructificación erecta, estipitada y ramificada, con aspecto de Clavaria TREMELLODENDRON (IX)

2 Fructificación nunca con aspecto de Clavaria 3 
3 Fructificación estipitada con píleo alargado, irregular o infundibuliforme

3 Fructificación sin estipe o subestipitada

4. Resupinada con himenio seco o ceroso

4. Efusa, lobada, girosa o cerebriforme, con himenio gelatinoso
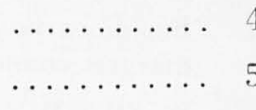

5 Resupinada, himenio seco, con basidios alargados-fusiformes, y oblicuamente septados PATOUILLARDINA (XI)

5 Resupinada, himenio seco o ceroso, liso, papilado o espinado; basidios globosos a ovoides, cruciado-septados

6 Girosa a cerbriforme, blanca cuando fresca; himenio con gleocistidios

DUCTIFERA (XII)

6 Sin gleocistidios

7 Lobada, girosa o cerebriforme, blanca o anaranjada cuando fresca; esporas subglobosas u ovoides TREMELLA (XIII)

7 Efusa a foliosa y subestipitada; nunca de color anaranjada; esporas alantoides EXIDIA (XIV)

8 Himenio liso, seco o ceroso-gelatinoso; sin gleocistidios SEBACINA (XV)

8 Himenio con gleocistidios

9 Himenio con hifas modificadas, formando papilas HETEROCHAETE (XVI)

9 Himenio sin papilas, liso o con espinitas

10 Himenio liso, nunca zonado .BOURDOTIA (XVII)

10 Himenio con espinitas o, si liso, marcado con zonas concéntricas EICHLERIELLA (XVIII)

\section{CLAVE DE LAS ESPECIES DE TREMELLA}

1 Basidiocarpo erecto, blanco cuando fresco y con lóbulos anastomosados $\ldots \ldots \ldots$. fuciformis

1 Basidiocarpo nunca de color blanco

2 Folioso, de color variable, en general marrón oscuro, nunca amarillo o anaranjado T. foliacea

2 Lobulado a cerebriforme, de color amarillentoanaranjado

$\ldots \ldots \ldots$. lutescens 
BOLETIN DE LA SOCIEDAD BOTANICA DE MEXICO, No. 29

\section{CLAVE DE LAS ESPECIES DE EXIDIA}

1 Basidiocarpo gris-blanco y aplanado, con pelos hialinos vsibles en el margen cuando seco

E. mexicana sp. nov.

1. Basidiocarpo sin pelos

2 Basidiocarpo efuso, blanquecino en estado fresco, con nódulos calcáreos visibles en el himenio cuando seco $\quad . . \ldots \ldots \ldots$. E. nucleata

2 Basidiocarpo con píleo erecto, marrón oscuro en estado fresco, negro al secar

........... recisa

\section{CLAVE DE LAS ESPECIES DE SEBACINA}

1 Basidiocarpo ceroso, esporas 10-13 x 4-5 micras S. adusta

1 Basidiocarpo seco, esporas $15-20 \times 7.9$ micras S. calcea

\section{CLAVE DE LAS ESPECIES DE HETEROCHAETE}

1 Basidiocarpo delgado (menos de 50 micras de grosor), esporas menos de 15 micras de largo H. shearii

1 Basidiocarpo más grueso (frecuentemente hasta 600 micras), esporas hasta 25 micras de largo H. livido-fusca

\section{CLAVE DE LAS ESPECIES DE EICHLERIELLA}

1 Basidiocarpos con margen blanco, himenio con espinitas ........... spinulosa

1 Basidiocarpos sin margen, himenio sin espinas ........... leveilliana

\section{DACRYMYCETACEAE}

(I) Calocera cornea (Fries) Loudon

Los basidiocarpos son hasta de $1 \mathrm{~cm}$. de altura y variables en su color que es amarillento a anaranjado. El himenio es anfígeno y las esporas son de 7.9 × 3.4 micras, 1-septadas.

Material estudiado: Cerro Cabezas, La Marquesa. Méx. MRO, TH, BL. 45. 1.VII-'62; San Cayetano, Méx., TH, MRO, BL 176, 12-VII-'62; San Cristóbal Las Casas, Chis., BL 136, 3-VIII-'62. 
(II) Dacryopinax spathularia (Schw.) Martin

El basidiocarpo es alargado en la parte superior, hasta $5 \mathrm{~mm}$. de ancho y con estipe delgado. El himenio es unilateral y las esporas son 8-11 x 4-5 micras.

Material estudiado: Necaxa, Pue., MRO, TH, GG, 29-X-48; Desierto de los Leones, D.F.. MRO, TH 83, 16-VII-'50; Baños La Primavera, Guadalajara, Jal., GG No. 2849, VII-'61; San Cristóbal Las Casas, Chis., BL 130, 3-VII-'62; Faldas del Nevado de Toluca, Méx., BL 265, 19-VIII-‘62.

Dacryopinax elegans (Berk \& Curt.) Martin. micras.

Distintamente pileado, estipe con pelos largos. Las esporas miden $13-17$ x $5-7$

Material estudiado: Catemaco, cerca de la Laguna, Ver., BL 112, 19-VII-'62; Teapa, Tab., BL 164, 22-VII-'62.

(III) Ditiola nuda Berk. \& Br.

= Dacryomitra nuda (Berk. \& Br.) Pat.

El basidiocarpo tiene aspecto de una Morchella minúscula de hasta 3-4 mm de altura. Las esporas son unitriseptadas, 10-13 x 4-5 micras.

Material estudiado: Teapa, Tab., BL 166, 22, VII-‘62.

(IV) Arrhytidia involuta (Schw.) Coker Lám. 1, figs. G-L.

En esta especie, el basidiocarpo es resupiando y efuso y tiene el aspecto de manchitas de color marrón oscuro. La textura es cerosa y las esporas miden 15-18 x 5-7 micras. Son uni-triseptadas.

Material estudiado: Faldas del Nevado de Toluca, Méx., BL 268, 19-VII-`62.

(V) Dacrymyces ellisii Coker

El basidiocarpo es fuertemente radicado en sl substrato, es convoluto, de color anaranjado cuando fresco y las esporas son 3-septadas, 10-13 x 5-7 micras. Kennedy (5), trata esta especie como una variedad de D. deliquescens.

Material estudiado: La Marquesa, D.F., TH, GG, BL 37, 38, 39, 24-VI-‘62; Cerro Cabezas, D.F., TH, BL 47, 1-VII-'62.

Dacrymyces dictyosporus Martin Lam. I, figs. A-F.

Esta especie, conocida antes sólo de Honduras (Martin 21), tiene un aspecto muy similar a $D$. palmatus, de la que difiere en las esporas muriformes que miden 18-26 x 7-9 micras y tienen de 2 hasta 6 o más septas longitudinales.

Material estudiado: San Cayetano, Méx., TH, BL 87, 12-VIII-`62; San Cristóbal Las Casas, Chis., BL 125, 3-VIII- 62.

Dacrymyces palmatus (Schw.) Bres. 
Los basidiocarpos son estipitados, hasta $6 \mathrm{~mm}$. de altura y con himeneo convoluto. Las esporas son 7 -septadas y miden 18-21 x 5-7 micras. La colección examinada ha perdido su color original anaranjado por haberse conservado en formol. pero la estructura microscópica es típica.

Material estudiado: Lagunas de Zempoala, Méx., A. Barrera VF 211, IX-55. Dacrymyces deliquescens (Merat) Duby

Los basidiocarpos, al secar, son apenas visibles, pero cuando frescos son de color amarillo-anaranjado, gelatinosos, convolutos y a veces subestipitados. Las esporas miden $11-15 \times 4-6$ micras.

Material estudiado: La Marquesa, Méx., MRO, TH, BL 34, 35, 24-VI-'62; Cerro Cabezas, Méx., Evangelina Pérez 65, 15 -VII-662.

Dacrymyces punctiformis Neuh.

Los basidiocarpos miden cerca de $1.2 \mathrm{~mm}$. en el estado seco y son de color ana. ranjado. Las esporas son $13-16$ x 4-4.5 micras.

Material estudiado: El Zarco, Méx., BL 46, 1-VIII-‘62; San Cristóbal Las Casas, Chis., BL 128, 4-VIII-'62.

\section{AURICULARIACEAE}

\section{(VI) Auricularia delicata (Fries) Henn.}

Se nota la característica diagnóstica de esta especie en las circunvoluciones del himenio que forman una red. Como indican Herrera y Guzmán (4), se emplea a veces en México como alimento. Las esporas de las especies de Auricularia son muy similares y miden más o menos 10-14 x 5-6 micras. Son unicelulares, cilíndri. cas o levemente curvadas.

Material estudiado: Tenejapa, Hgo., MRO, TH 80, 4-XI-48; Ajenjibre, Pue., MRO, TH 66, 76, 79, 81, '51-52; Ojo de Agua, Ver., MRO, TH 66, 20-III-49; San Agustín Loxicha, Oax., GG No. SF 1132, IX-'57; Tuxtepec, Oax., MRO, TH 16, 15-IX-47; Villa Juárez, Pue., F. Márquez 12, 12-IX-60; Catemaco, cerca de la laguna, Ver., BL 118, 19-VII-62; Cosamaloapan, Ver., BL 119. 17-VII-620; Teapa. Tab., BL 160 161, 162, 22-VII-‘62; San Cristóbal las Casas, Chis., BL 127, 3 . VIII. 62 .

Auricularia auricula (Hook.) Underw.

El basidiocarpo en corte transversal carece de médula. En su aspecto superficial se puede confundir con A. fuscosuccinea, especialmente cuando seco. Los detalles referentes a la organización interna de las especies de Auricularia se encuentran en otro trabajo del autor (8). 
Material estudiado: Desierto de los Leones, D.F., TH 69, 23-VII-‘50; Ajenjibre, Pue., MRO, TH 74, 20-V-552; Salazar, Méx., O. Ruiz C. 18, 1o-I-60; Cascada de Diamantes, Iztaccíhuatl, Méx., T. Wantabe 15, 24-VII-“60.

Auricularia mesenterica Pers.

El basidiocarpo es notable por su abhimenio zonado y piloso y el himenio que tiene venas conspicuas. Es de textura rígida, no gelatinosa.

Material estudiado: Palo Bolero, Mor., GG No. OF 2316, 26-VII-‘60; Tuxtepec, Oax., GG No. SF 2795, 30-X-'60; Cerca de Taxco, Gro., BL 210, 16-VIII-'62.

Auricularia polytricha (Mont.) Sacc.

En estado fresco el basidiocarpo es cartilagíneo-gelatinoso, y tiene pelos largos en un himenio no zonado (Lowy, 9).

Material estudiado: San Luis Potosí, S.L.P., I. Roa P. 5, II-‘60; Minatitlán, Ver., No. 6, 24-II-60 ( $\sin$ nombre del colector); Ojo de Agua, Ver., MRO, TH 25, 3-XI-48; Lagunas de Zempoala, Méx., MRO 27, 14-III-60; Acapulco, Gro., MRO, TH 30, IX-52; Tuxtepec, Oax., MRO, TH 32, 12-IX-49; Cuautla, Mor., I. López No. OF 2172, 16-VIII-'59; El Chico, Hgo., MRO, TH 67, 15-IX'50; Ajenjibre, Pue., MRO, TH 85, 21-I-52; Faldas del Volcán Tacaná, Chis., GG No. SF 522 b, IX·56; Teapa, Tab., BL 165, 22-V1I-`62; Cosamaloapan, Ver., BL 114, 119, 16-17. VII.'62; San Andrés Tuxtla, Ver., BL 102, 19-VII-‘62.

(VII) Platygloea carnea Pat.

El basidiocarpo pequeño y efuso es levemente convoluto cuando mojado, y en el espécimen estudiado, 5-6 mm. de largo. Es de color marrón-rojizo cuando seco. Las esporas son 8-10 × 5-6 micras.

Material estudiado: Oaxaca, Oax., A. J. Sharp 17793, VIII-‘35.

\section{TREMELLACEAE}

(VIII) Pseudohydnum gelatinosum (Fries) Karst.

$=$ Tremellodon gelatinosum Fries

El himenio espinoso es diagnóstico del género. El píleo que mide hasta $5 \mathrm{~cm}$. de largo es duro-gelatinoso y blanquecino cuando fresco y se pone rígido y de color más oscuro al secar. Las espinas son hasta $5 \mathrm{~mm}$. de largo y las esporas subglobosas, 5.8 micras en diámetro.

Material etudiado: La Marquesa, Méx., GG y E. Hidalgo No. OF 29, 22-VI-‘54; La Marquesa, Méx., GG y F. Medellín No. OF 270, I-X-55; Salazar, Méx., No. 23, 5.VIII-56 ( $\sin$ nombre del colector); Bosque de Chapultepec, Hgo., GG No. GH 61206, 20-IX-61; Puerto Buenos Aires, Hgo., GG No. 61258, 21. IX·661. 


\section{(IX) Tremellodendron candidum (Fries) Atk.}

Los basidiocarpos forman un grupo ramificado cerca de $6 \mathrm{~cm}$. de altura y 3 de ancho, con los ápices más o menos libres. El himenio anfígeno es limitado a la parte inferior de la fructificación y las esporas miden 12-15 x 8-10 micras.

Material estudiado: Morelos, J. N. Rose, 15-VIII-“06 [espécimen en los Na. tional Fungus Collections, Beltsville, Md., identificado como T. merismatoides (Schw.) Burt.|

(X) Phlogiotis helvelloides (Fries) Martin Lam. 2, A-F.

= Gyrocephalus rufus Bref.

Las fructificaciones tienen hasta $5 \mathrm{~cm}$. de altura, un estipe de $3.5 \mathrm{~mm}$. de diá. metro y un píleo ancho (más o menos de $1 \mathrm{~cm}$ ). El himenio es inferior y la esporas son 10-12 x 5-6 micras. Cuando seco es de color marrón-rojizo. Se encuentran en el suelo bajo coníferas.

Material estudiado: Faldas de Popocatépetl, Méx., GG No. VF 237, 28-VIII-55; Lagunas de Zempoala, Méx., GG No. VF 1851, 14-VIII-55; La Marquesa, Méx., GG No. VF 2696, I-X-55; Salazar, Mléx., TH 43, 28-VIII-55; Ajusco, D. F., A. Hernández C., No. VTR 1200, 23-IX-557; Paso Cortés, Popocatépetl, Méx., R. Singer 9. 21-VII-‘57; Cerro Cabezas, La Marquesa, Méx., TH 24, 30-IX-‘56.

(XI) Patouillardina cinerea Bres.

El basidiocarpo es efuso, hasta $10 \times 3 \mathrm{~cm}$. y tiene el aspecto de un Corticium. Los basidios son notables por su forma fusiforme que miden alrededor de $40 \times 10$ micras. Las esporas son 10-12 × 4-6 micras.

Material estudiado: Jalapa, Ver., C.L. Smith 213, 1894-1896. (Espécimen en el herbario de la Universidad de Iowa).

\section{(XII) Ductifera pululahuana (Pat.) Donk Lám. 3, fig. A.}

= Exidia alba (Lloyd) Burt

El basidiocarpo es gelatinoso, convoluto y blanquecino cuando fresco, duro y de color levemente marrón cuando seco. Los gleocistidios en sl subhimenio son ahun. dantes y largos, hasta $100 \times 10$ micras. Las esporas miden $10-12 \times 5-6$ micras.

Material estudiado: Tres Marías, Cuernavaca, Mor., GG No. OF 2022, 20-VII. 57; Cuernavaca, Mor., R. Heim No. OF 2144, 9-VIII-57; Bosque Chapultepec, D. F.. Maxine y Doris Lowy, BL 36, 5-VII-`62.

(XIII) Tremella fuciformis Berk.

La fructificación es erecta, gelatinosa, lobada, hasta $2-3 \mathrm{~cm}$. de altura, $5 \mathrm{~cm}$. 0 más de ancho. Es blanca en estado fresco y al secarse se pone de color levemente marrón. Las esporas son subglobosas a ovoides, 6-9 micras de diámetro. 
Material estudiado: Región de El Salto, Dgo., GG No. GH 61129, 18-IX.‘61. Tremella foliacea Fries. Lám. 3, fig. B.

Esta especie tiene basidiocarpos que se cuentan entre los más grandes de los Tremellales. - La colección encontrada fue de $6 \mathrm{~cm}$. de altura y $8 \mathrm{~cm}$. de ancho, con lóbulos numerosos y foliosos, de color marrón oscuro cuando seco. Las esporas son ovoides y miden $12-15 \times 10-13$ micras.

Material estudiado: San Cristóbal las Casas, Chis., BL 139, 4-VIII-662.

\section{Tremella lutescens Fries}

\section{$=T$. mesenterica Fries}

Los basidiocarpos son lobados, erecios, $2-3 \mathrm{~cm}$. de altura y de color amarilloanaranjado cuando frescos. Las esporas son subglobosas u ovoides, 10-15 x 8-12 micras. $T$. lutescens y $T$. mesenterica, en mi opiniór, representan una sola especie variable. Looney (7) y otros han considerado este problema.

Material estudiado: Desierto de los Leones, D. F., MRO, TH 71, 10-VII-49; Xilitla, S.L.P., GG y J. Rzedoswki No. NF 2966, VII-49; Pedregal de San Angel, Cerros de Xitla Conejo, D. F., 26-VI-52; La Marquesa, Méx., GG y F. Medellín No. VF 269 a, 1-X-55; Salazar, Cerro de la Campana, Méx., MRO, TH, Martha Zenteno 10, 21-VIII-60; Faldas del Popocatépetl, Méx., GG. G. Hernández C. No. VF 3035, 8-VIII-‘62; San Cayetano, Méx., BL 90, 12-VIII-‘62.

\section{(XIV) Exidia nucleata (Schw.) Burt}

Se encuentran los basidiocarpos en forma de manchas blancas gelatinosas e irregulares de $1.2 \mathrm{~cm}$. de largo. Las esporas son alantoides y miden 10-12 x 4-5 micras.

Material estudiado: Teapa, Tab., BL 207, 22-VII-62; Lagunas de Zempoala, Méx., GG No. XF 3014 a, 3-VIII- 62 .

Exidia recisa Fries

Los basidiocarpos, hasta $2 \mathrm{~cm}$. de largo, son pileados y gelatinosos.

Las hifas tienen numerosas hebillas y las esporas miden 12-14 x 4-5 micras.

Material estudiado: Omitlán, Hgo., B. Dutary 13, 17-VII-60; Zacualtipán, Hgo., TH 14, 16-I-61; La Marquesa, Méx., MRO, TH, BL 33, 24-VI-662; San Cayetano, Méx., BL 95 A, 12-VIII-‘62.

Exidia mexicana sp. nov. Lam. 2, figs. G-K.

Fructificatio in humido gelatinosa, alba, coalescens deinde effuso; in sicco cornea, sordide alba, circa $2.3 \mathrm{~cm}$. lata, cum margine conspicuo, hyalino-hispida; hyme- 
nio papillosa, minutissime rugulosa; hyphae nodosae, 2.5 - 3.5 u diam., probasidia subglobosa vel ovata, deinde cruciatim septata, (10-) 12 - 16 (-18) X (8-) 10 - 14 micras; sterigmata cilindracea, circa $60-75$ × 3-5 micras; basidiosporae ovoidea, hyalinae, aseptatae, (13-) 14-16 x (7-) 910.5 micras; per repetitionem germinantes vel promycelium promittentes.

Ad ligna demortua Arctostaphylos crescit. La Marquesa, Méx., Evangelina Pérez, GG, TH, BL 40, 24-VI-‘62; TYPUS; La Marquesa, Méx., BL 44, 1-VII-‘62.

Los basidiocarpos en el estado fresco son blancos y gelatinosos con márgenes conspicuos. En su origen se presentan como pequeñas pústulas en la corteza, que crecen en diámetro y al final se ponen efusos. Cuando secos, cambian a un color más oscuro. En el margen se notan los pelos blancos que tienen un tamaño de hasta $1 \mathrm{~mm}$. Las papilas del himenio no son numerosas. En el subhimenio se encuentran (no muy regularmente), nódulos blancos amorfos de un material cristalino. Las hifas del basidiocarpo son 2.5 - 3.5 micras de diámetro y tienen hebillas. También tienen hebillas las hifas que llevan los basidios. (Lám. 2, fig. I). Los probasidios son sul. globosos u ovoides (10-) 12-16 (-18) X (8-) 10-14 micras con 2 septas en forma de cruz; los esterigmas (epibasidios) miden hasta $75 \times 3-5$ micras. Las basidiosporas son ovoides, hialinas y unicelulares, (13-) 14-16 x (7-) 9-10 micras y germinan por repetición o por la producción de un promicelio. La nueva especie está más relacio. nada con Exidia hispidula Lowy, colectada en Louisiana, EE.UU. Lowy (11).

\section{(XV) Sebacina adusta Burt}

Cuando seco, el basidiocarpo es efuso y de color marrón oscuro. Se puede identificar por las esporas que miden 10-13 x 4-5 micras.

Material estudiådo: Cuernavaca, Mor., W.A. \& E.L. Murril No. 370, 24.27, XII-'09; Cosamaloapan, Ver., BL 119 A, 16-VII-62.

\section{Sebacina calcea (Pers.) Bres.}

El basidiocarpo es delgado (cerca de 100-125 micras), con gránulos amorfos en el subhimenio. Los diarioparafisos son notables por su variación y son ramificados o sencillos. Wells (27) incluye esta especie en el género Exidiopsis.

Material estudiado: Cuernavaca, Mor., W.A. \& E.L. Murril, 24-27-XII-‘09.

\section{(XVI) Heterochaete shearii (Burt) Burt}

Las papilas estériles que se proyectan del himenio constituyen la característica diagnóstica del género. En esta especie, las esporas miden 11-15 x 5-6 micras. Para detalles sobre la estructura de los basidiocarpos se debiera consultar el trabajo de Bodman (1).

Material estudiado: Lagunas de Zempoala, Méx., GG No. XF 3015, 3-VI.62; Cerro Cabezas, Méx., MRO, TH, BL 45 A, 1-VII-`62. 


\section{LOWY, ESTUDIO SOBRE TREMELLALES DE MEXICO}

Heterochaete livido-fusca Pat.

Se distingue esta especie de la anterior por el basidiocarpo más grueso y las esporas largas que son $20-25 \times 8-11$ micras.

Material estudiado: San Andrés Tuxtla, Ver., BL 105, 19-VII-662.

(XVII) Bourdotia cinerea (Bres.) Bourd. \& Galz.

Bourdotia se diferencia de Sebacina por los gleocistidios en el subhimenio. En B. cinerea, éstos son variables, pero miden alrededor de $50 \times 8$ micras. Las esporas son de $10-12$ × 5.7 micras.

Material estudiado: Motzorongo, cerca de Córdoba, Ver., W.A. \& E.L. Murrill No. 986 (TIPO), 15-I-‘10; San Cayetano, Méx., TH, MRO, BL 171, 12-VIII-‘62.

(XVIII) Eichleriella spinulosa (Berk. \& Curt.) Burt

El basidiocarpo tiene el aspecto de un Radulum (Hydnaceae) y estaba incluído en este género originalmente. Pero los basidios son tetraseptados y las esporas alantoides de $15-20 \times 6-8$ micras. Las espinitas en el himenio son hasta $5 \mathrm{~mm}$. de largo.

Material estudiado: Catemaco, Ver., BL 103, 19-VII-‘62.

Eichleriella leveilliana (Berk. \& Curt.) Burt

El basidiocarpo es aplanado y a veces se notan en el himenio zonas concéntricas. Las esporas miden $15-17$ × 5-7 micras.

Material estudiado: Cerro Cabezas, Méx., TH, BL 43 A, 1-VII.662; Cosamaloapan, Ver., BL 110, 16-VII-'62.

\section{EXPLICACION DE LAMINAS}

LAMINA 1. A-F, Dacrymyces dictyosporus. A, Probasidio con dicarioparafisis; B, C, Basidios en distintos estados de desarrollo; D, Dictiospora característica de la especie; E, Basidio maduro con 2 esterigmas (epibasidios); las esporas se prenden en los ápices; F, Dibujo del basidiocarpo en perfil, X 2; G - L, Arrhytidia involuta. G, Probasidio con dicaryoparafisis; H, I, J, Basidios en distintos estados de desarrollo; J, Basidio maduro; K, Dos esporas, una 3-septada, la otra inmadura; L, Dibujo del basidiocarpo, visto de arriba, X 3.

LAMINA 2. A-F, Phlogiotis helvelloides. A, Basidiocarpo X 1.5; B, Probasidio; C, E, Basidios en distintos estados de desarrollo; comúnmente, en esta especie, se forman sólo dos células en el basidio. Se notan los esterigmas (epibasidios) alargados, que terminan en un punto agudo cuando están maduras; D, Dos esporas maduras; F, Hifa con hebilla; G-K, Exidia mexicana sp. nov. G, Probasidio que es cruciado-septado: H, Basidiospora madura; I, Probasidio en perfil (septado) con 
dicarioparafisis; J, Basidio con 4 esterigmas; K, Probasidio con 2 esterigmas en pro. ceso de formación.

LAMINA 3. A, Ductifera pululahuana apx. X 1; B, Tremella foliacea apx. X 2/3.

\section{SUMMARY}

The study of some jelly fungi of Mexico in this paper includes 33 species distributed in 17 genera. A new species: Exidia mexicana is described. Taxonomic keys for the mexican fungi of this group are proposed for the first time.

\section{REFERENCIAS BIBLIOGRAFICAS}

1. Bodman, M.C. 1949. The genus Heterochaete in the United States. Mycologia 41: 527-536.

2. Donk, M.A. 1954. A note on sterigmata in general. Bothalia 6: 301-302.

3. . . N . 1956. Notes on resupinate Hymenomycetes II. The tulasnelloid fungi. Reinwardtia 3: $363-379$.

4. Herrera, T. y G. Guzmán. 1962. Taxonomía y ecología de los principales hongos comes. tibles de diversos lugares de México. Anales Inst. Biol. 32 (1, 2): 33-135.

5. Kennedy, L.L. 1958. The genus Dacrymyces. Mycologia 50: 896-915.

6. Linder, D.H. 1940. Evolution of the basidium and its relation to the terminology of the basidium. Mycologia 32: 419-447.

7. Looney, A.M. 1933. A morphological study of certain species of Tremella. Univ. Iowa Stud. Nat. Hist. 15: 17-33.

8. Lowy, B. 1951. A morphological basis for classifying the species of Auricularia. Myco. logia 43: $351-358$.

9. $\quad \ldots \ldots \ldots \ldots$ 1952. The genus Auricularia. Mycologia 4.t: 656-692.

10. ....... 1954. A new species of Platygloea from Louisiana. Mycologia 46: 100-104.

11. ........1957. A new Exidia. Mycologia 19: 899-902.

12. ....... 1959. New or noteworthy Tremellales from Bolivia. Mycologia 51: 840-850.

13. ........1962. Contribución al conocimiento de los Tremellales de la Argentina. Lilloa. (En prensa).

14. Martin, G. W. 1938. The morphology of the basidium. Amer. Jour. Bot. 25: 682-685.

15. ........ 1938. New or noteworthy fungi from Panamá and Colombia. II. Mycologia 30: $431-441$.

16. ....... 1941. New or noteworthy tropical fungi. Lloydia t: 262-269.

17. ....... New or noteworthy tropical fungi. III. Lloydia $7: 67-80$.

18. ....... 1948. New or noteworthy tropical fungi. IV. Lloydia 11: 111-123.

19. ........ 1952. Revision of the North Central Tremellales. Univ. Iowa Stud. Nat. Hist. 19: $1-122$.

20. ........ The tulasnelloid fungi and their bearing on basidial terminology. Brittonia 9: $25-30$.

21. ........ 1958. A new species of Dacrymyces from Honduras. Mycologia 50: 939-941.

22. Moeller, A. 1895. Protobasidiomyceten. Vol. 8. Botanische Mittheilungen aus den Tropen.

23. Neuhoff, W. 1924. Zytologie und systematische Stellung der Auriculariaceen und Tremel. laceen. Bot. Arch. 8: 250-297.

24. Patouillard, N. et G. Lagerheim. 1893. Champignons de l'Equateur. Bull. Soc. Myc. Fr. 9: 124-165.

25. Rogers, D.P. 1934. The basidium. Univ. Iowa Stud. Nat. Hist. 16: 160-183.

26. Talbot, P.H.B. 1954. Micromorphology of Lower Basidiomycetes. Bothalia 6: 249-299.

27. Wells, K. 1961. Studies of some Tremellaceae. IV. Exidiopsis. Mycologia 53: 317-370.

28. Viegas, A.P. 1945. Algunos fungos do Brasil. V, VI. Basidiomycetos. Auriculariales. Bra. gantia 5: 197-212; Dacryomycetaceae - Tremellaceae. 239-251. 

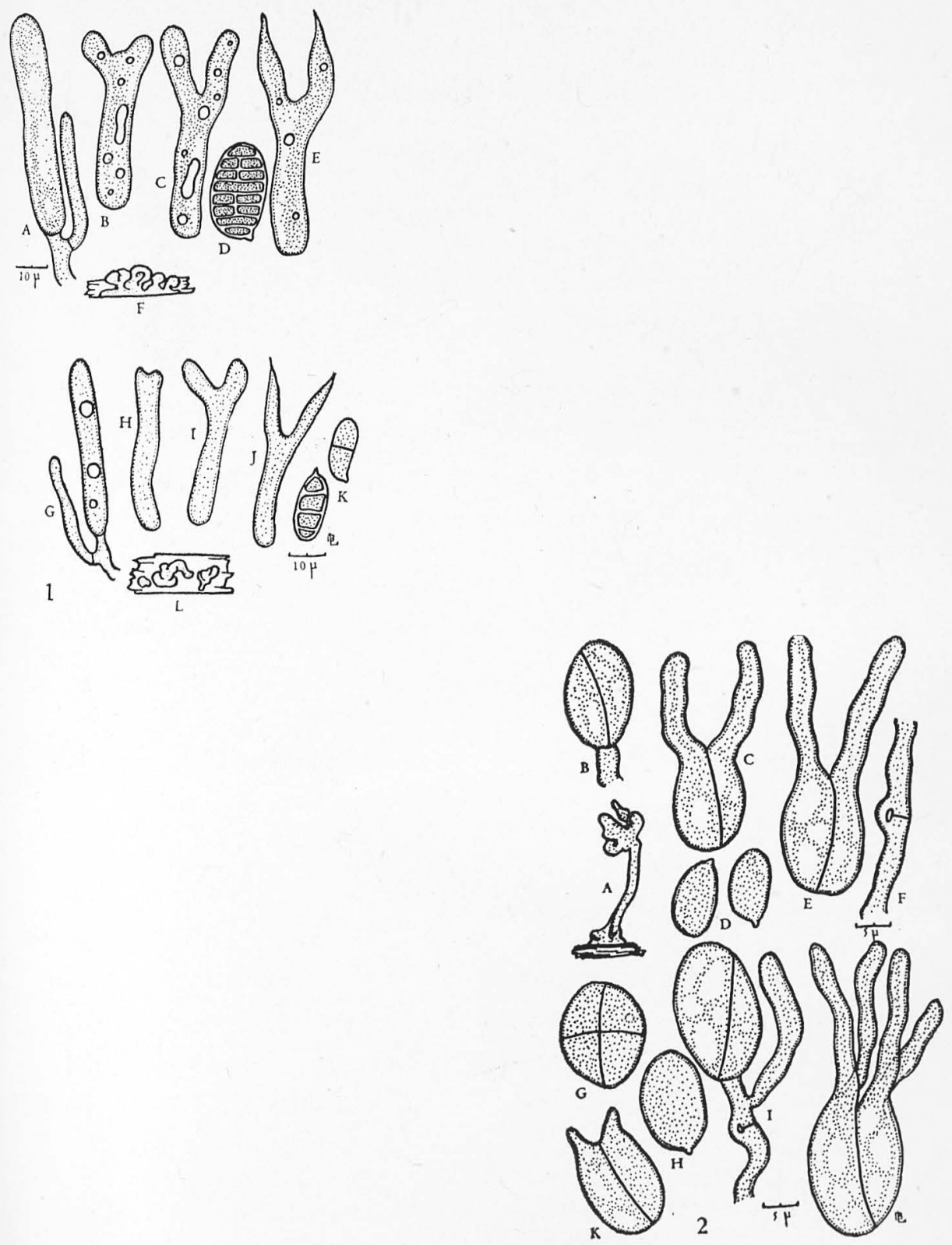